\title{
Ornithine and Tryptophan Analogs as Efficient Polycations for Short Interference RNA Delivery to Tumor Cells
}

\author{
Yusuke Sato, Hiroto Hatakeyama, and Hideyoshi Harashima* \\ Faculty of Pharmaceutical Sciences, Hokkaido University; Kita 12, Nishi 6, Kita-ku, Sapporo 060-0812, Japan. \\ Received February 9, 2010; accepted April 10, 2010; published online April 20, 2010
}

\begin{abstract}
The delivery of nucleic acids to cancer cells represents a potentially useful strategy. Previously, we developed a multifunctional envelope-type nano device (MEND) for the efficient delivery of plasmid DNA. In addition, we successfully delivered short interference RNA (siRNA) into cytoplasm using a MEND which contains siRNA particles that were produced using stearyl octaarginine (STR-R8). In the present study, to achieve further gene silencing activity compared with STR-R8, various additional polycations were screened. We used protamine and 10 different polypeptides containing random sequence of basic amino acids. The ability of these polycations to form nano particles with siRNA were evaluated by measuring the size and zeta-potential of produced nano particles, and as a consequence, 6 of the polycations were selected for further evaluation. We then prepared MENDs containing the particles. The lipid composition of the MEND consisted of dioleoylphosphatidyl ethanolamine (DOPE)/phosphatidic acid (PA) (7/2). For cellular uptake and endosomal escape, the MEND was modified with PPD (polyethylene glycol (PEG)-peptide-DOPE), STR-R8 and GALA, pH-sensitive fusogenic peptide. The resulting MEND had a diameter of $120-170 \mathrm{~nm}$ and a zeta-potential of $15-25 \mathrm{mV}$. The MEND was transfected into HeLa cells stably expressing luciferase and the silencing activity of the polycations was compared. Most of the polycations failed to knockdown luciferase activity. However, the polypeptide containing ornithine and tryptophan (Orn/Trp) induced a higher knockdown than STR-R8. In addition, Orn/Trp induced a silencing effect at lower doses than STR-R8, as evidenced by dose-response data. In conclusion, the findings suggest that Orn/Trp is a superior polycation to STR-R8 for siRNA delivery.
\end{abstract}

Key words non-viral delivery system; short interference RNA; ornithine; tryptophan; multifunctional envelope-type nano device

RNA interference (RNAi), initiated by small interfering RNA (siRNA) is a promising strategy for curing a variety of human diseases, including cancer., ${ }^{1,2}$ To obtain an efficient knockdown of the target gene, siRNA must be delivered to the cytosol of the target cells. Many groups have developed various types of carrier systems for siRNA delivery, such as lipoplexes and polyplexes. ${ }^{3-5)}$ We recently reported on the development of a novel gene and nucleic acid delivery system, a multi functional envelope-type nano device (MEND) ${ }^{6,7)}$ A MEND consists of a core that contains nucleic acids complexed with polycations, which is then covered by a lipid envelope. The complexed core minimizes the space taken up by the nucleic acids and thus improves packaging efficiency, and the resulting core/shell structure protects the nucleic acids from degradation by enzymes. The physical properties such as size and activity of the complexed nucleic acids are influenced by the polycations. For example, the gene expression efficiency of a MEND containing a condensed plasmid DNA(pDNA) core comprised of protamine is greater than that for a MEND with a condensed pDNA core comprised of poly-L-lysin (PLL) or stearyl occtaarginine (STR-R8). ${ }^{8,9)}$ On the other hand, STR-R8 is favorable for efficient siRNA delivery and gene silencing compared with protamine and PLL. ${ }^{10)}$ We previously reported on the development of a MEND prepared with dioleoylphosphatidyl ethanolamine (DOPE) and phosphatidic acid (PA) as a siRNA delivery system for tumors. ${ }^{11}$ Modification of the lipid envelope with STR-R8, $\mathrm{pH}$-sensitive fusogenic peptide GALA in the form of cholesteryl-GALA (Chol-GALA), and cleavable polyethylene glycol (PEG)-lipid (PEG-peptideDOPE: PPD) in response to matrix metalloproteinase (MMP) successfully facilitated the delivery of siRNA delivery to tumor cells as the result of an increase in both cellular uptake and endosomal escape. In the present study, to further enhance silencing activity of the MEND we attempted to improve the siRNA core via the use of a more superior complexer for siRNA delivery than STR-R8. We tested protamine and 10 types of random sequence polypeptides containing basic amino acids such as lysine (Lys), arginine (Arg), or ornithine (Orn). We first screened suitable polycations for siRNA complexation, as assessed by the size of resulting complex. A MEND was then prepared with the successfully formed siRNA complex. The silencing ability of the polycations was estimated by the knockdown of luciferase knockdown in HeLa cells.

\section{MATERIALS AND METHODS}

Materials Anti-luciferase siRNA (21-mer, 5'-GCGCUGCUGGUGCCAACCCTT-3', 5' -GGGUUGGCACCAGCAGCAGCGCTT-3') and anti-green fluorescent protein (GFP) siRNA (5'-GCUGACCCUGAAGUUCAUCTT-3', 5'-GAUGAACUUCAGGGUCAGCTT-3') were obtained from Thermo Electron GmbH (Ulm, Germany). DOPE and rhodamine-DOPE (Rho-DOPE) were purchased from AVANTI Polar Lipids (Alabaster, AL, U.S.A.). PA, poly(ornithine and tryptophan) (Orn/Trp, 4:1,n=155, M.W. 30000), poly(lysine and tryptophan) (Lys/Trp, 4:1, $n=187$, M.W. 38000), poly(arginine and tryptophan) (Arg/Trp, 4:1, $n=155$, M.W. 29700), poly-L-histidine (His, $n=42$, M.W. 5800), poly-L-lysine (Lys, $n=135$, M.W. 28200), poly-L-arginine (Arg, $n=184$, M.W. 35500), poly(ornithine and serine) (Orn/Ser, $3: 1, n=164$, M.W. 27500), poly(arginine, proline and threonine) (Arg/Pro/Thr, 6:3:1, $n=116$, M.W. 18100), poly- 
(lysine and phenylalanine) (Lys/Phe, $1: 1, n=199$, M.W. 35400), and poly(lysine and tyrosine) (Lys/Tyr, $4: 1, n=123$, M.W. 24600) were purchased from Sigma-Aldrich (St. Louis, MO, U.S.A.). Protamine sulfate was obtained from Calbiochem (Ishikari, Japan). PPD and Chol-GALA were synthesized as described previously. ${ }^{12,13)}$ Dulbecco's modified Eagle's medium (DMEM) and fetal bovine serum (FBS) were purchased from Invitrogen (Carlsbad, CA, U.S.A.). Diethylpyrocarbonate (DEPC)-treated water and G418 were obtained from Nacalai Tesque (Kyoto, Japan). Luciferase assay reagents and reporter lysis buffer were obtained from Promega (Madison, WI, U.S.A.). HeLa human cervical carcinoma cells were obtained from the RIKEN Cell Bank (Tsukuba, Japan). All other chemicals were commercially available reagent-grade products.

Cell Culture HeLa cells stably expressing luciferase (HeLa-luc) were cultured in cell-culture dishes (Corning) containing DMEM supplemented with $10 \%$ FBS, penicillin (100 U/ml), streptomycin $(100 \mu \mathrm{g} / \mathrm{ml}), \mathrm{G} 418(0.4 \mathrm{mg} / \mathrm{ml})$ at $37^{\circ} \mathrm{C}$ in an atmosphere of $5 \% \mathrm{CO}_{2}$ and $95 \%$ humidity.

Preparation of siRNA Complexes and MEND To prepare the siRNA/polycation complex, siRNA in DEPC-treated water was complexed with the polycation in DEPC-treated water, at a nitrogen/phosphate $(\mathrm{N} / \mathrm{P})$ ratio of 3.0 in $240 \mu \mathrm{l}$. A lipid film was formed by the evaporation of a chloroform solution containing DOPE, PA, PPD and Chol-GALA (118 nmol total lipids in $7: 2: 0.5: 0.1$ molar ratio). The siRNA/polycation complex was then applied to the lipid film, followed by incubation for $10 \mathrm{~min}$ at room temperature to hydrate the lipids. To coat the siRNA/polycation complex core with the lipid, the lipid film was then sonicated for approximately $1 \mathrm{~min}$ in a bath-type sonicator. An STR-R8 solution (10 mol\% of lipids) was added to the suspension to attach STR-R8 to the envelope, and the mixture was then incubated for $30 \mathrm{~min}$ at room temperature. The average diameter and zeta-potential of the siRNA/polycation core and MEND were determined using a Zetasizer Nano ZS ZEN3600 (MALVERN Instrument, Worchestershire, U.K.).

Gene Silencing Effect of MEND To examine the silencing effect of the MEND, $4 \times 10^{4} \mathrm{HeLa}$-luc cells were seeded in a 24-well dish $1 \mathrm{~d}$ prior to transfection. The MEND, containing the indicated dose of siRNA, was added to $0.25 \mathrm{ml}$ of DMEM containing 10\% FBS, followed by incubation at $37^{\circ} \mathrm{C}$ for $3 \mathrm{~h}$. A $0.75 \mathrm{ml}$ aliquot of DMEM containing $10 \%$ FBS was added to the cells, followed by incubation for an additional $21 \mathrm{~h}$. The cells were then washed with $0.5 \mathrm{ml}$ of phosphate buffered saline (PBS) and lysed with reporter lysis buffer. Luciferase activity in the cell lysate was then measured using a luminometer (Luminescencer-PSN, ATTO, Japan). Protein concentrations were determined with a BCA Protein Assay Kit (Pierce, Rockford, IL, U.S.A.). Luciferase activity was expressed as relative light units (RLU) per mg of protein. The silencing effect was calculated as a percentage using the following equation:

$$
\text { silencing effect }(\%)=\left(1-\frac{T E_{\text {anti-luc }}}{T E_{\text {anti-GFP }}}\right) \times 100
$$

where $T E_{\text {anti-luc }}$ and $T E_{\text {anti-GFP }}$ represent luciferase gene expression after transfection with either anti-luciferase siRNA or anti-GFP siRNA, respectively.
Cellular Uptake of MEND To evaluate the amount of cellular uptake of MEND, $4 \times 10^{4} \mathrm{HeLa}-\mathrm{luc}$ cells were seeded in a 24-well dish $1 \mathrm{~d}$ prior to transfection. The MEND labeled with Rho-DOPE ( $7 \mathrm{~mol} \%$ of total lipid) was added to $0.25 \mathrm{ml}$ of DMEM containing $10 \% \mathrm{FBS}$, followed by incubation at $37^{\circ} \mathrm{C}$ for $3 \mathrm{~h}$. The cells were then washed with $0.5 \mathrm{ml}$ of PBS and lysed with reporter lysis buffer, followed by centrifugation at $12000 \mathrm{rpm}$, for $5 \mathrm{~min}$, at $4{ }^{\circ} \mathrm{C}$ to remove debris. Fluorescence intensity of rhodamine in the supernatant was determined using FP-750 Spectrofluorometer (JAS Co., Tokyo, Japan) (Excitation: 557 nm, Emission: $589 \mathrm{~nm}$ ). Protein concentration was determined with a BCA Protein Assay Kit (Pierce, Rockford, IL, U.S.A.). Cellular uptake of MEND was expressed as fluorescence intensity per mg of protein.

Statistic Analysis Comparisons between multiple treatments were made using one-way analysis of variance (ANOVA), followed by the Student-Newman-Keuls test. Pair-wise comparisons between treatments were made using a two-tail Student $t$-test. A $p$-value of $<0.05$ was considered significant.

\section{RESULTS AND DISCUSSION}

In initial experiment, we evaluated the complexation of siRNA with polycations. From the view point of tumor targeting via the enhanced permeability and retention (EPR) effect and intracellular trafficking, controlling size is a critical factor. Therefore, the feasible diameter of siRNA core was assumed to be less than $100 \mathrm{~nm}$. Among the 12 polycations we examined, 7 polycations including STR-R8 formed a siRNA core complex with a diameter of $60-80 \mathrm{~nm}$ and retained a positively charge, as shown in Table 1 . As reported previously, STR-R8 is a suitable complex agent for siRNA as compared with poly-L-Lysin and protamine, presumably because the hydrophobic stearyl moiety plays an important role in forming a complex with siRNA as small particles. ${ }^{10)}$ With the exception of polyArg, polypeptides composed of basic amino acids and hydrophobic amino acids all resulted in the formation of small size particles $(<100 \mathrm{~nm})$, but polypeptides composed of only basic amino acids (His and Lys), or basic amino acids and hydrophilic amino acids (Orn/Ser and

Table 1. Characters of siRNA Core and MEND

\begin{tabular}{|c|c|c|c|c|c|}
\hline \multirow[b]{2}{*}{ Polycation } & \multirow[b]{2}{*}{$\begin{array}{c}\text { Molar } \\
\text { ratio }\end{array}$} & \multicolumn{2}{|c|}{ Core } & \multicolumn{2}{|c|}{ MEND } \\
\hline & & $\begin{array}{l}\text { Diameter } \\
(\mathrm{nm})\end{array}$ & $\begin{array}{l}\text { Zeta- } \\
\text { potential } \\
(\mathrm{mV})\end{array}$ & $\begin{array}{l}\text { Diameter } \\
(\mathrm{nm})\end{array}$ & $\begin{array}{c}\text { Zeta- } \\
\text { potential } \\
(\mathrm{mV})\end{array}$ \\
\hline STR-R8 & - & $59 \pm 6$ & $15 \pm 1$ & $119 \pm 16$ & $18 \pm 6$ \\
\hline Orn/Trp & $4: 1$ & $61 \pm 1$ & $5 \pm 2$ & $133 \pm 24$ & $18 \pm 5$ \\
\hline Lys/Trp & $4: 1$ & $80 \pm 11$ & $11 \pm 4$ & $137 \pm 48$ & $23 \pm 6$ \\
\hline Arg/Trp & $4: 1$ & $69 \pm 7$ & $16 \pm 2$ & $135 \pm 21$ & $18 \pm 8$ \\
\hline His & - & fail & - & - & - \\
\hline Lys & - & fail & - & - & - \\
\hline Arg & - & $74 \pm 14$ & $7 \pm 4$ & $164 \pm 51$ & $15 \pm 3$ \\
\hline Orn/Ser & $3: 1$ & fail & - & - & - \\
\hline Arg/Pro/Thr & $6: 3: 1$ & $83 \pm 8$ & $5 \pm 4$ & $132 \pm 24$ & $19 \pm 3$ \\
\hline Lys/Phe & $1: 1$ & $77 \pm 10$ & $19 \pm 5$ & $168 \pm 51$ & $15 \pm 6$ \\
\hline Lys/Tyr & $4: 1$ & fail & - & - & - \\
\hline Protamine & - & fail & - & - & - \\
\hline
\end{tabular}

Data are mean \pm S.D. of at least 3 different preparations. 


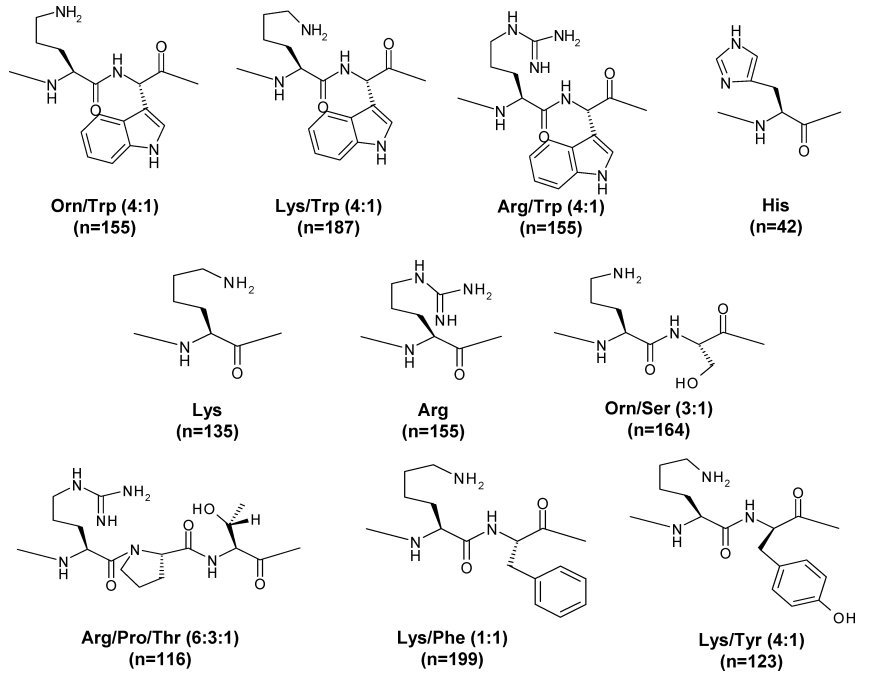

Fig. 1. The Structure of Polycations Used in This Study

Fractions in parentheses denote the molar ratio of each amino acid. " $n$ " indicates the total number of amino acids residues.

Lys/Tyr) failed to form cores with small diameters. These observations are in agreement with previous results. Therefore, it seems reasonable to conclude that a hydrophobic moiety is required for the formation of siRNA particles. Then, we prepared MEND with successfully formed siRNA complex. In the absence of PPD, the size of the MEND was larger $(>200$ $\mathrm{nm}$ ), while the diameter of a PPD-modified MEND was less, around $120-170 \mathrm{~nm}$, because steric hinderance by the PEG moiety inhibited the interaction and fusion of each lipid envelope, leading to the formation of aggregates. In the case of the MEND modified with STR-R8 on the surface of lipid envelope after packaging of the siRNA core complex, the zetapotential was positive $(15-25 \mathrm{mV})$. The characteristics of the MENDs were largely unaffected by polycations.

We examined the effect of variations in the polycation used on the silencing activity of prepared MEND. As shown in Fig. 2a, the MEND in which siRNA core contained Lys/ Trp, Arg/Trp, Arg/Pro/Thr, Lys/Phe, or Arg showed minor or negligible silencing activity at a dose of $0.4 \mu \mathrm{g}$ siRNA. However, the silencing activity of the MEND prepared using Orn/Trp was superior to that of the MEND prepared using STR-R8. To achieve silencing activity mediated by siRNA, MEND must be taken up by target cells via endocytosis, followed by endosomal escape. The expression of MMP-2 in the supernatant of HeLa-luc was approximately $20 \mathrm{ng} / \mathrm{ml}$, which is sufficient to trigger the cleavage of PEG. ${ }^{12)}$ Therefore, PPD could be cleaved in response to MMP secreted from HeLa cells, which resulted in that R8 exerted their ability to internalize MEND into cells via endocytosis pathway. In the endosomal compartment, GALA induces fusion of the lipid envelope with the endosomal membrane, followed by the release of the siRNA core into cytosol. Finally, the siRNA core must be de-complexed, which allows siRNA to be free and to become loaded on RNA induced silencing complex (RISC). As shown in Fig. 2b, cellular uptake of MEND was independent of the kinds of polycation. The extent of endosomal release of the siRNA core into the cytosol mediated by GALA might be comparable and independently of the specific polycation used. Therefore, the differential in a
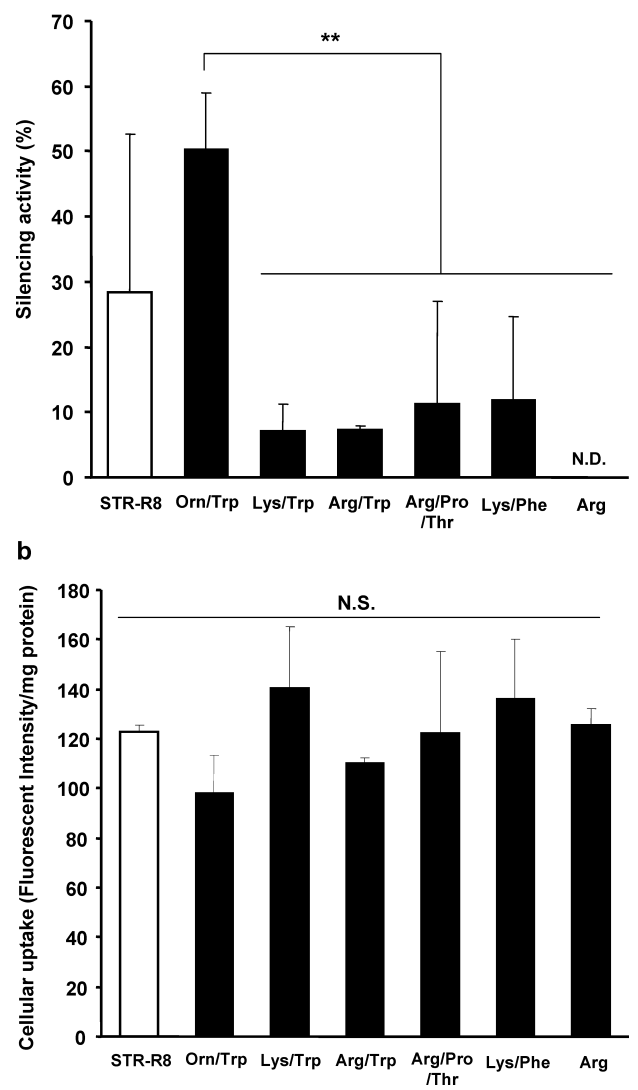

Fig. 2. Comparison of the Silencing Activity and Cellular Uptake of MEND with Various Types of Polycations

a) MENDs ( $0.4 \mu \mathrm{g}$ siRNA/well; $0.12 \mu \mathrm{m}$ siRNA) containing various types of polycations were transfected into HeLa-luc $\left(4 \times 10^{4}\right.$ cells/well), and luciferase activity was measured $24 \mathrm{~h}$ after transfection. The RNAi effect was calculated by normalization to untreated cells. The RNAi effect is expressed as the mean \pm S.D. $(n=3)$. $* * p<0.01$. N.D.: Not detected. b) MENDs ( $0.4 \mu \mathrm{g}$ siRNA/well) labeled with Rho-DOPE containing various types of polycations were transfected into HeLa-luc $\left(4 \times 10^{4}\right.$ cells/well), and fluorescence intensity was measured $3 \mathrm{~h}$ after transfection. Cellular uptake is expressed as the mean \pm S.D. $(n=3)$. N.S.: No significant difference.

silencing activity could be due to the ability of the polycation to release siRNA from the core particle. Despite the same hydrophobic amino acid (Trp) and molar ratio of basic amino acids and hydrophobic amino acids $(4: 1)$ in comparison with Orn/Trp, Lys/Trp, and Arg/Trp, only Orn/Trp knocked down the target gene. It is possible that a minor difference of structure around the amine group which generates the cationic charge alters rate of release siRNA, as shown in Fig. 1. Finally, the dose dependency of STR-R 8 and Orn/Trp on silencing activity was further evaluated. The silencing activity reached a plateau at doses of $0.4 \mu \mathrm{g}$ or larger, as shown in Fig. 3a. The MEND with Orn/Trp showed an enhanced silencing activity at lower doses of siRNA within $0.1-$ $0.2 \mu \mathrm{g}$ compared with STR-R8 (Fig. 3a). On the other hand, at higher doses of siRNA within $0.4-0.6 \mu \mathrm{g}$, the activity of the MEND with STR-R8 was equal to that with Orn/Trp. At all doses, no cytotoxicity was observed compared to nontreatment, as shown in Fig. 3b. Therefore, the knockdown would be saturated at higher doses. Further study regarding intracellular trafficking, or the release process is clearly needed to completely understand the mechanism of the enhanced activity of Orn/Trp compared with STR-R8. It is important that, from the view point of side effect and economi- 

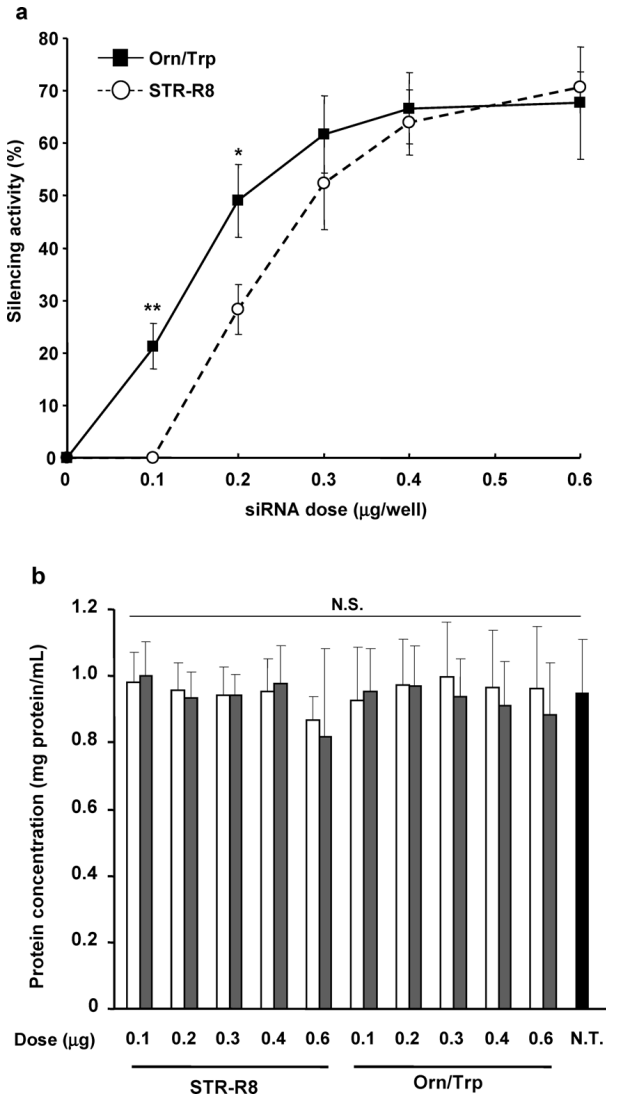

Fig. 3. Dose Dependency of MEND with STR-R8 or Orn/Trp on Silencing Activity

a) MEND with STR-R8 or Onr/Trp core was transfected into HeLa-luc $\left(4 \times 10^{4}\right.$ cells/well) at doses of $0.1,0.2,0.3,0.4$ and $0.6 \mu \mathrm{g}$ siRNA/well $(0.03,0.06,0.09,0.12$, $0.18 \mu \mathrm{M}$ siRNA), and luciferase activity was measured $24 \mathrm{~h}$ after transfection. The RNAi effect was calculated by normalization to cells treated with non-specific (antiGFP) siRNA. The RNAi effect is expressed as the mean \pm S.D. $(n=3) . * p<0.05$, $* * p<0.01$. b) Protein concentration is expressed as the mean \pm S.D. $(n=3)$. Open bars: anti-luciferase siRNA, gray bars: anti-GFP siRNA, black bar; untreated.

cal efficiency, higher knockdown activity should be obtained using less dose of siRNA. In addition, siRNAs delivered to tumor tissue could be diluted faster than that in normal tissue due to division of the tumor cells unlike normal cells, result- ing in less activity of siRNA. Based on the findings reported herein, it can be concluded that Orn/Trp is a more suitable siRNA complexer than STR-R8 for siRNA medicine against cancer. These results of our study suggest that optimization of the sequential steps of intracellular trafficking from cellular uptake to siRNA release into the cytosol is useful process that results in improved siRNA delivery.

Acknowledgments This work was financially supported by Grants-in-Aid for Young Scientists (Start-up) from the Ministry of Education, Culture, Sports, Science and Technology of Japan, and by Grants-in-Aid for Scientific Research on Priority Areas from the Japan Society for the Promotion of Science. The authors also wish to thank Dr. Milton Feather for his helpful advice in writing the English manuscript.

\section{REFERENCES}

1) Kim D. H., Rossi J. J., Nat. Rev. Genet., 8, 173-184 (2007).

2) Fougerolles A., Vornlocher H. P., Maraganore J., Liberman J., Nat. Rev. Drug Discov., 6, 443 - 453 (2007).

3) Li S. D., Chono S., Hunag L., J. Controlled Release, 126, 77-84 (2008).

4) Tietze N., Pelisek J., Philipp A., Roed W., Merdan T., Tarcha P., Ogris M., Wagner E., Oligonucleotides, 18, 161-174 (2008).

5) Leng Q., Scaria P., Lu P., Woodle M. C., Mixson A. J., Cancer Gene Ther, 15, 485-495 (2008).

6) Kogure K., Moriguchi R., Sasaki K., Ueno M., Futaki S., Harashima H., J. Controlled Release, 98, 317-323 (2004).

7) Kogure K., Akita H., Yamada Y., Harashima H., Adv. Drug Deliv. Rev., 60, 559-571 (2008).

8) Masuda T., Akita H., Harashima H., FEBS Lett., 579, 2143-2148 (2005).

9) Moriguchi R., Kogure K., Akita H., Futaki S., Miyagishi M., Taira K., Harashima H., Int. J. Pharm., 301, 277-285 (2005).

10) Nakamura Y., Kogure K., Futaki S., Harashima H., J. Controlled Release, 119, 360-367 (2007).

11) Sakurai Y., Hatakeyama H., Hidetaka A., Oishi M., Nagasaki Y., Futaki S., Harashima H., Biol. Pharm. Bull., 32, 928 - 932 (2009).

12) Hatakeyama H., Akita H., Kogure K., Oishi M., Nagasaki Y., Kihira Y., Ueno M., Kobayashi H., Kikuchi H., Harashima H., Gene Ther. 14, 68-77 (2007).

13) Futaki S., Ishikawa T., Niwa M., Kitagawa K., Yagami T., Bioorg. Med. Chem., 5, 1883-1891 (1997). 